ARTICLE

Received 23 Jul 2014 | Accepted 7 Jan 2015 | Published 17 Feb 2015 DOl: 10.1038/ncomms7227

\title{
Primitive macrophages control HSPC mobilization and definitive haematopoiesis
}

\author{
Jana Travnickova ${ }^{1,2,3, \star}$, Vanessa Tran Chau ${ }^{1,2,3,4,5, \star}$, Emmanuelle Julien $6,7,8$, Julio Mateos-Langerak ${ }^{9}$, \\ Catherine Gonzalez ${ }^{1,2,3}$, Etienne Lelièvre ${ }^{1,2,3,10}$, Georges Lutfalla ${ }^{1,2,3}$, Manuela Tavian ${ }^{6,7,8}$ \& Karima Kissa $a^{1,2,3,10}$
}

In vertebrates, haematopoietic stem/progenitor cells (HSPCs) first emerge in the aorta-gonadmesonephros (AGM) before colonizing transitory and subsequently definitive haematopoietic organs allowing haematopoiesis throughout adult life. Here we identify an unexpected primitive macrophage population accumulated in the dorsal mesenteric mesoderm surrounding the dorsal aorta of the human embryo and study its function in the transparent zebrafish embryo. Our study reveals dynamic interactions occurring between the HSPCs and primitive macrophages in the AGM. Specific chemical and inducible genetic depletion of macrophages or inhibition of matrix metalloproteinases (Mmps) leads to an accumulation of HSPCs in the AGM and a decrease in the colonization of haematopoietic organs. Finally, in vivo zymography demonstrates the function of primitive macrophages in extracellular matrix degradation, which allows HSPC migration through the AGM stroma, their intravasation, leading to the colonization of haematopoietic organs and the establishment of definitive haematopoiesis.

\footnotetext{
${ }^{1}$ CNRS UMR 5235, F-34095 Montpellier, France. ${ }^{2}$ Universite Montpellier 2, Dynamique des Interactions Membranaires Normales et Pathologiques, F-34095 Montpellier, France. ${ }^{3}$ Universite Montpellier 1, F-34000 Montpellier, France. ${ }^{4}$ Institut Pasteur, Unité Macrophages et Développement de I'Immunité, Département de Biologie du Développement, F-75015 Paris, France. ${ }^{5}$ CNRS, URA2578, F-75015 Paris, France. ${ }^{6}$ INSERM UMR_S949, F-67000 Strasbourg, France. ${ }^{7}$ Universite de Strasbourg, F-67000 Strasbourg, France. ${ }^{8}$ Etablissement Français du Sang-Alsace, F-67000 Strasbourg, France. ${ }^{9}$ Montpellier RIO Imaging, F-34396 Montpellier, France. ${ }^{10}$ INSERM, DIMNP, F-34095 Montpellier, France. ${ }^{*}$ These authors contributed equally to this work. Correspondence and requests for materials should be addressed to K.K. (email: karima.kissa@univ-montp2.fr).
} 
B lood cells are continuously produced from self-renewing progenitors with multi-lineage differentiation potential, called haematopoietic stem/progenitor cells (HSPCs). In vertebrates, haematopoiesis occurs as two waves. The 'primitive' wave produces only myelo-erythroid cells, whereas the 'definitive' wave gives rise to $\mathrm{HSPCs}^{1-4}$. In zebrafish, from 16 hours post fertilization (h.p.f.), primitive haematopoietic cells produce macrophages and neutrophils that initiate innate immunity ${ }^{5-7}$. Zebrafish first HSPCs appear in the aorta-gonad-mesonephros (AGM), between the dorsal aorta (DA) and the posterior cardinal vein (PCV) by 30 h.p.f. (refs 3,8) where they start to express cd41 marker ${ }^{9}$. These HSPCs originate from aortic endothelial cells ${ }^{10,11}$ through a process called endothelial haematopoietic transition or $\mathrm{EHT}^{11}$. Live imaging of mouse embryo sections ${ }^{12}$, cell sorting and differentiation experiments in human embryo have shown haemogenic endothelial cells to be at the immediate origin of haematopoietic cell clusters sprouting from the ventral side of the aorta ${ }^{13}$, which suggest that this process is conserved throughout evolution. We have previously documented that zebrafish HSPCs migrate from the AGM, enter the circulation and colonize the caudal haematopoietic tissue (CHT), analogous to the mammalian fetal liver. There they proliferate before colonizing the definitive haematopoietic organs, thymus and kidney ${ }^{9}$. HSPCs emerging between 30 and 55 h.p.f. give rise to all mature blood cell populations, including the second wave of macrophages.

Macrophages from the first and second waves of haematopoiesis play important roles in development, tissue homeostasis ${ }^{14}$, innate and adaptive immunity and wound healing. In adults, they are also involved in the pathologies including chronic diseases ${ }^{15}$ and cancer metastasis ${ }^{16,17}$.

Macrophage-related processes are associated with their ability to migrate throughout the body and remodel surrounding stroma ${ }^{18}$ by degrading the extracellular matrix (ECM) via the secretion of matrix metalloproteinases (MMPs for human and Mmps for zebrafish) and cathepsins ${ }^{19}$. MMPs have been shown to play important roles in tissue remodelling, or pathological processes ${ }^{20}$, especially MMP-2, 9 and 13. In zebrafish embryos, Mmp-9 and 13a are expressed by myeloid cells from 19h.p.f. (refs 21,22).

In this study, we imaged the accumulation of primitive macrophages in the AGM of both human and zebrafish embryos and analysed their interactions with emerging HSPCs. We demonstrated that primitive macrophages play a major role in the establishment of definitive haematopoiesis by permitting HSPC mobilization and colonization of haematopoietic organs via Mmps secretion and ECM remodelling.

\section{Results}

Macrophages accumulate in AGM during HSPC emergence. Human HSPCs emerge between the 27th and the 40th day of development in the AGM as clusters of $\mathrm{CD} 34+$ $\mathrm{CD} 45+$ cells adhering to the ventral wall of the $\mathrm{DA}^{23}$. Immunohistochemistry in human embryos at 34 days of development $(n=2)$ showed an intriguing accumulation of $\mathrm{CD} 68+$ macrophages in the mesenchyme beneath the ventral part of the $\mathrm{CD} 34+$ aortic endothelium (Fig. $1 \mathrm{a}-\mathrm{a}^{\prime \prime}, \mathrm{b}-\mathrm{b}^{\prime \prime}$ ) and in the HSPC containing intra-aortic clusters (Fig. $\left.1 b^{\prime}, b^{\prime \prime}\right)$. The number of macrophages in these locations, increased between 26 and 37 days of gestation, that is, in concomitance with the emergence of HSPCs (Supplementary Fig. $1 ; n=3$ ).

We took advantage of zebrafish embryo remarkable optical properties to study the possible role of these macrophages during definitive haematopoiesis.

In vivo imaging of $\operatorname{Tg}(c d 41: e G F P / / m p e g 1: m C h e r r y)$ zebrafish embryos at 43 h.p.f. (green HSPCs and red macrophages) showed a similar accumulation of primitive macrophages along the entire length of the trunk in the direct vicinity of the ventral wall of the DA (Fig. 1c).

Macrophage quantification confirmed their tissue-restricted accumulation in zebrafish AGM compared to surrounding tissues (Fig. 1d, $n=20$ ). Interestingly, this accumulation was concomitant with $\mathrm{HSPC} / \mathrm{cd} 41: \mathrm{eGFP}+$ emergence in the AGM between 30 and 55 h.p.f. (ref. 11).

Macrophages actively patrol in the AGM. To assess if macrophages play a role in HSPC emergence, we imaged the behaviour of HSPCs and macrophages in Tg(cd41:eGFP//mpeg1:mCherry) zebrafish embryos at 43 h.p.f. (Fig. 1e-l). As previously described, endothelial cells from the ventral wall of the aorta first bend then egress into the subaortic space to become free HSPCs ${ }^{11}$. At this stage, we observed that macrophages (Fig. 1f) continuously patrolled between the DA and the PCV, and intimately interacted with emerging HSPCs/cd41:eGFP + (Fig. 1e-l, Supplementary Movies 1 and 2). Live imaging of $T g(c d 41: e G F P / / m p e g 1: m$ Cherry $)$ zebrafish embryo at 55 h.p.f. showed a macrophage/ mpegl:mCherry + migrating in the AGM around an HSPC/ cd41:eGFP + during $32 \mathrm{~min}$, before joining the PCV (Supplementary Movie 2, time code 00:56 to 1:00). This HSPC/ cd41:eGFP + would then join the PCV following the same route (Supplementary Movie 2). A quantification of 112 HSPCs/ cd41:eGFP + (from 12 embryos) in the AGM revealed that $95.6 \%$ of them directly interacted with macrophages suggesting that macrophages could play an important role in emergence of HSPCs in the AGM.

\footnotetext{
Figure 1 | Accumulation of macrophages in the AGM at the onset of definitive haematopoiesis. (a-b') Immunostained transverse sections of AGM region in 34-day human embryos $(n=2)$. ( $\left(\mathbf{a}, \mathbf{a}^{\prime}, \mathbf{b}, \mathbf{b}^{\prime \prime}\right)$ CD34 labels position of aortic endothelium (arrowhead) and HSPCs ( $\mathbf{a}^{\prime}$, asterisks and arrows). ( $\left.\mathbf{a}^{\prime}-\mathbf{a}^{\prime}\right)$

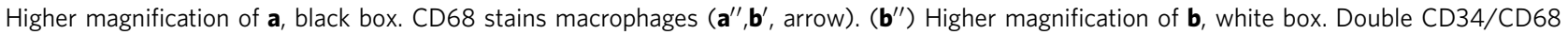
immunofluorescence shows accumulation of macrophages (arrow and empty arrow), respectively, in the ventral wall of the DA and in HSPCs intra-aortic clusters (red arrow). (c) Maximum projection of confocal z-stack acquisitions in a Tg(cd41:eGFP//mpeg1:mCherry) zebrafish embryo at 43 h.p.f. (10 $\mu \mathrm{m}$ depth); bright field is merged with green and red fluorescence. HSPCs/cd41:eGFP + (arrow) and macrophages/mpeg1:mCherry (arrowhead) accumulate between the DA and the PCV. (d) Graph representing the specific accumulation of macrophages in the AGM during EHT compared with macrophages present in the intersomitic vessels and in pronephric ducts area. Data are shown as average \pm s.e.m, $n=20$ (from two independent experiments). $P$ values are calculated for the difference between AGM and both intersomitic vessels and pronephric duct area at 40,45 and 50 h.p.f. and for the difference of macrophage number in the AGM between 30 and 40 h.p.f. (underlined asterisks). ${ }^{\star \star \star} P<0.001$ (Kruskal-Wallis test for the whole evaluation and Student's t-test followed by Holm's multiple comparison for individual stages). (e-I) Time-lapse confocal imaging of Tg(cd41:eGFP// mpeg1:mCherry) zebrafish embryo at 43 h.p.f. (higher magnification of $\mathbf{c}$, black box). (f-j) A macrophage (arrowhead) actively patrols in the AGM around a $\mathrm{HSPC} / \mathrm{cd} 41: \mathrm{eGFP}+$ (arrow) for 20 min, before heading toward another HSPC/cd41:eGFP + (k,I). CV, cardinal vein; DA, dorsal aorta; ISV, intersomitic vessels; Mn, mesonephros; Nc, notochord; NT, neural tube; PCV, posterior cardinal vein; Pd, pronephric ducts; Ye, yolk extension. Scale bars, $50 \mu \mathrm{m}$ $\left(\mathbf{a}, \mathbf{b}^{\prime}, \mathbf{b}^{\prime \prime}\right), 25 \mu \mathrm{m}\left(\mathbf{a}^{\prime}, \mathbf{a}^{\prime \prime}, \mathbf{c}, \mathbf{e}-\mathbf{I}\right)$, and $100 \mu \mathrm{m}(\mathbf{b})$. See also Supplementary Fig. 1 and Supplementary Movies 1 and 2.
} 
Macrophages are essential for definitive haematopoiesis. To characterize their role in HSPC emergence, we chemically and genetically depleted primitive macrophages before or during definitive haematopoiesis at 25 and 45 h.p.f., respectively (before maturation of the second wave of macrophages).

Liposome encapsulated clodronate (L-clodronate) has been widely used to specifically induce the death of phagocytic macrophages in vitro and in vivo in mice and recently in zebrafish $^{24,25}$. We first set about testing its efficiency, toxicity and specificity. Injection of $5 \mathrm{nl}$ of L-clodronate stock suspension into the vein was sufficient for thorough macrophage depletion in embryos between 25 and 96h.p.f. (Supplementary Fig. 2b, $n=200$ ), compared with control embryos injected with PBS liposomes (L-PBS; Supplementary Fig. 2a, white arrows, $n=25$ ). The fate of macrophages in the AGM and in the CHT was examined during $18 \mathrm{~h}$ after L-clodronate injection. This
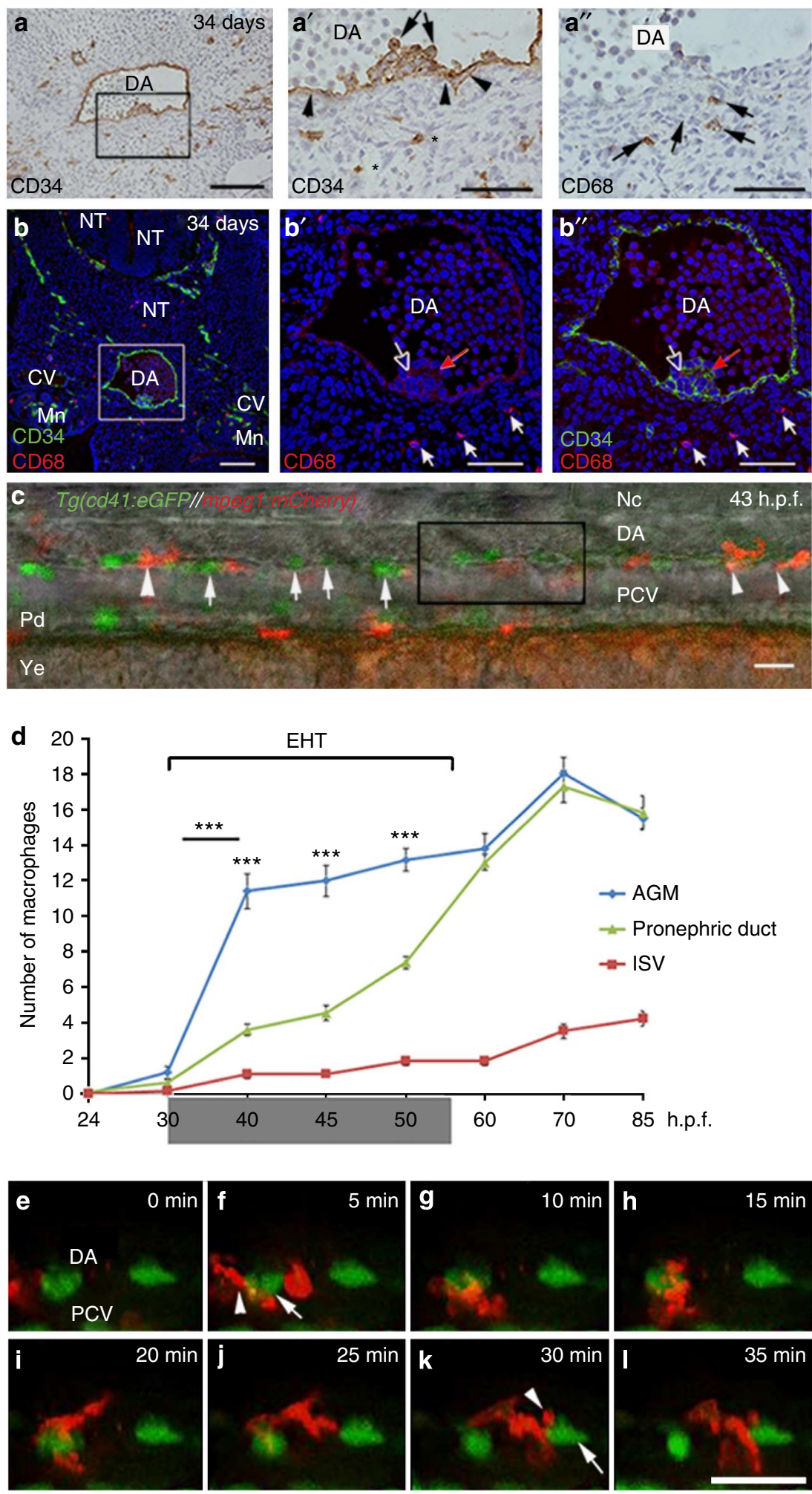
revealed the steady decrease to complete disappearance of macrophages while passing through vacuolization and fragmentation (Supplementary Fig. 2g). No toxic effect was noticed on blood vessel organization (Supplementary Fig. 2c,d, $\mathrm{Tg}$ (kdrl:caax:mCherry), $n=15)$ nor on the evolution (Supplementary Fig. 2h), differentiation and function of primitive wave neutrophils ${ }^{5}$, the other myeloid cell population present in the embryo at this stage of development (Supplementary Fig. 2e,f, arrow, $T g(m p x: e G F P), n=35)$.

To evaluate the effect of macrophage depletion on HSPC emergence (Fig. 2a-d), we injected L-clodronate in $\mathrm{Tg}(c d 41: e G F P)$ embryos at 25 h.p.f. and observed that macrophage depletion did not prevent HSPCs/cd41:eGFP + emergence in the AGM (Fig. 2b versus a), but led instead to their accumulation at 48 h.p.f. (Fig. 2 n, $+60 \%, n=20$ ). Whole-mount in situ hybridization (WISH) of $c$-myb confirmed the unaffected HSPC emergence in the AGM (Fig. 2d versus c). By contrast, macrophage depletion led to a very substantial decrease in the number of HSPCs/cd41:eGFP + colonizing the CHT (Fig. 2g,h versus e,f and $n ;-89 \%, n=28)$ and the thymus at 72 h.p.f. (Fig. 2l, versus $\mathrm{k}$ and $\mathrm{n} ;-91 \%, n=28$ ), while injecting L-clodronate at 45 h.p.f. (Fig. 2i) caused less marked effects ( $-72 \%$ in CHT: Fig. 2 j, $n, n=28$; and $-50 \%$ in the thymus: Fig. $2 \mathrm{~m}, \mathrm{n}, n=22$ ). The partial colonization observed after late L-clodronate injections was likely due to HSPCs that had already left the AGM before macrophage depletion. It further proved that L-clodronate was not toxic for HSPCs (Fig. 2j).

To fully validate the effect of macrophage depletion on haematopoietic organ colonization, we set inducible nitroreductase genetic depletion of macrophages ${ }^{26}$ as an alternate
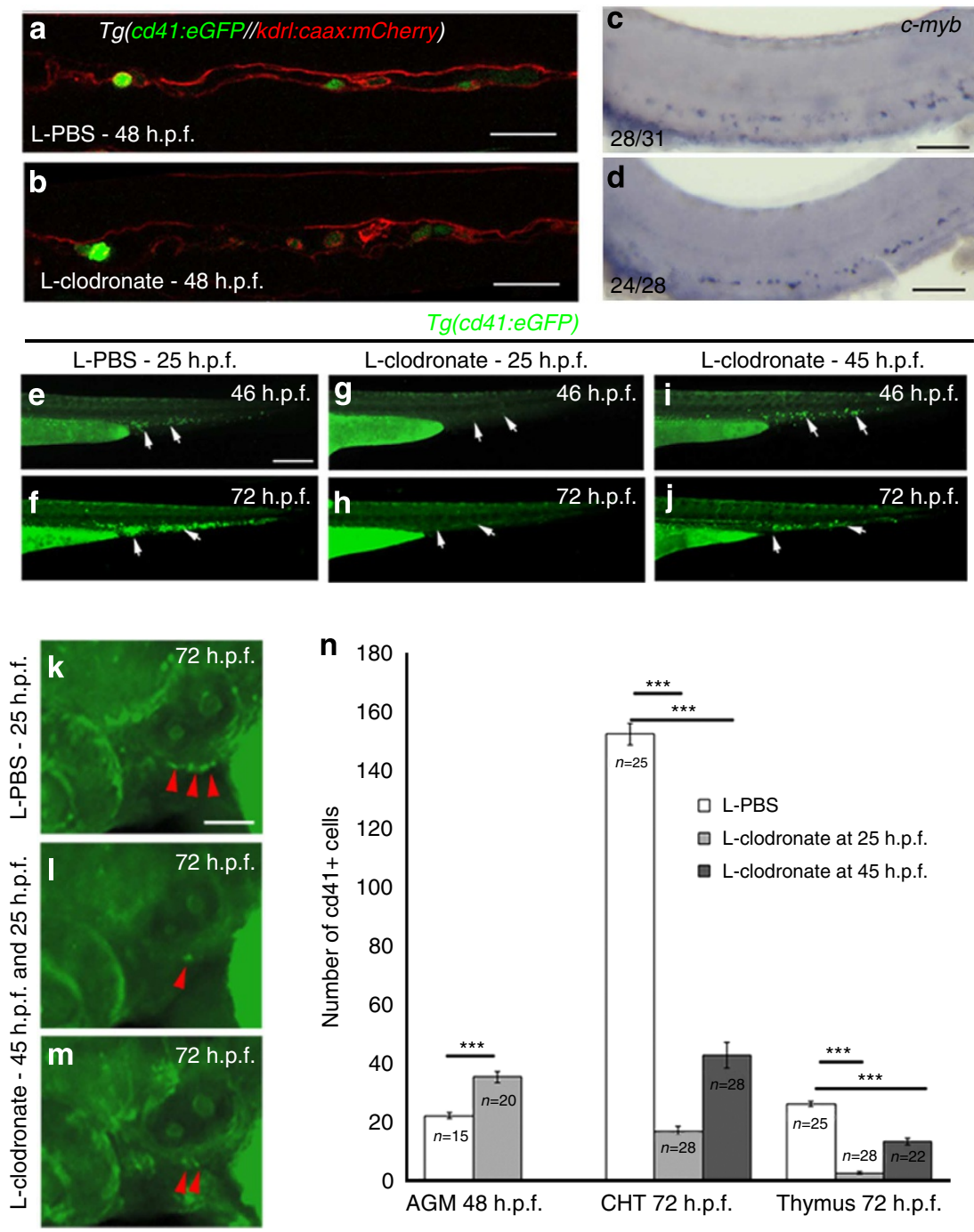

Figure 2 | Depletion of primitive macrophages reduces $\mathbf{C H T}$ and thymus colonization. (a,b) Macrophage depletion by L-clodronate injection at $25 \mathrm{~h}$.p.f. does not affect HSPC/cd41:eGFP + emergence in the AGM (48 h.p.f.). (c-d) WISH of c-myb shows normal HSPC emergence in the AGM after macrophage depletion $(N=21 \pm 0.72, n=15)$ compared with control embryos $(N=14.9 \pm 0.51, n=15 ; P<0.001$; Wilcoxon test). $N$ stands for the number of $c$-myb cells per embryo, $n$ for the number of embryos. (e-m) Macrophage depletion by L-clodronate injection at 25 or 45 h.p.f. decreases HSPC/ cd41:eGFP colonization at 46 and 72 h.p.f. of the CHT ( $\mathbf{g}-\mathbf{j}$, white arrow) and thymus (I-m, red arrowhead) compared with control injected with L-PBS $(\mathbf{e}, \mathbf{f}, \mathbf{k})$. (n) Macrophage depletion by L-clodronate injection at 25 h.p.f. causes HSPC/cd41:eGFP + accumulation in the AGM at 48 h.p.f. (left column, $+60 \%$ ), while injection at 25 or 45 h.p.f. substantially reduces HSPC/cd41:eGFP + colonization of the CHT at 72 h.p.f. ( -89 and $-72 \%$, respectively) and thymus colonization at 72 h.p.f. ( -91 and $-50 \%$, respectively) compared with the control injected with L-PBS. Data are shown as average \pm s.e.m. ${ }^{\star \star \star \star} P<0.001$ (Wilcoxon test), $n=$ number of embryos of minimal three independent experiments. Scale bars, $30 \mu \mathrm{m}(\mathbf{a}, \mathbf{b}), 100 \mu \mathrm{m}(\mathbf{c}, \mathbf{d}, \mathbf{k}-\mathbf{m})$ and $250 \mu \mathrm{m}$ (e-j). See also Supplementary Fig. 2 . 
experimental approach. Expression of E.coli nitroreductase under the control of mpeg1 promoter allows the inducible tissue specific and reversible depletion of macrophages by soaking embryos in the prodrug metronidazole. Similar to L-clodronate, metronidazole macrophage depleted embryos (Fig. $3 b$ versus a) exhibited an impaired CHT colonization by HSPCs/cd41:eGFP + (Fig. $3 b^{\prime}$ versus $\mathrm{a}^{\prime},-68 \%$ at 60 h.p.f., Fig. $3 c, n=24$ ) as well as an accumulation of HSPCs/cd41:eGFP in the AGM (Supplementary Fig. $3 a,+71 \%$ at 60 h.p.f., $n=11$ ). Macrophages still present after $42 \mathrm{~h}$ of metronidazole treatment (Fig. $3 \mathrm{~b}$ ) result either from partial depletion or delayed depletion or both and most likely explain the partial CHT colonization by HSPC compared with embryos injected with L-clodronate (Fig. $3 b^{\prime}$ and Fig. 2g,h).

As the genetic depletion is reversible by removing metronidazole from the medium, we measured macrophage reappearance during the 2 days following prodrug withdrawal (Fig. $3 \mathrm{~d}-\mathrm{g}$ ) and its effect on haematopoiesis in the CHT (Fig. $3 \mathrm{~d}, \mathrm{e}^{\prime}, \mathrm{f}^{\prime}, \mathrm{g}^{\prime}$ ). Indeed the number of HSPCs/cd41:eGFP + accumulated in the CHT was directly correlated with both the reappearance of macrophages in embryos (Fig. 3d and Supplementary Fig. 3b) and the decrease of the HSPCs/ cd41:eGFP accumulation in the AGM (Supplementary Fig. 3a).

Altogether these results demonstrated the crucial role of macrophages in haematopoietic organ colonization.

Mmps allow HSPC intravasation then organ colonization. Various proteases, especially MMP-2 and -9 that share high similarity in protein sequence with their zebrafish orthologs (90 and $73 \%$, respectively) ${ }^{21,27}$ have been shown to be associated with ECM degradation and cell intravasation mediated by
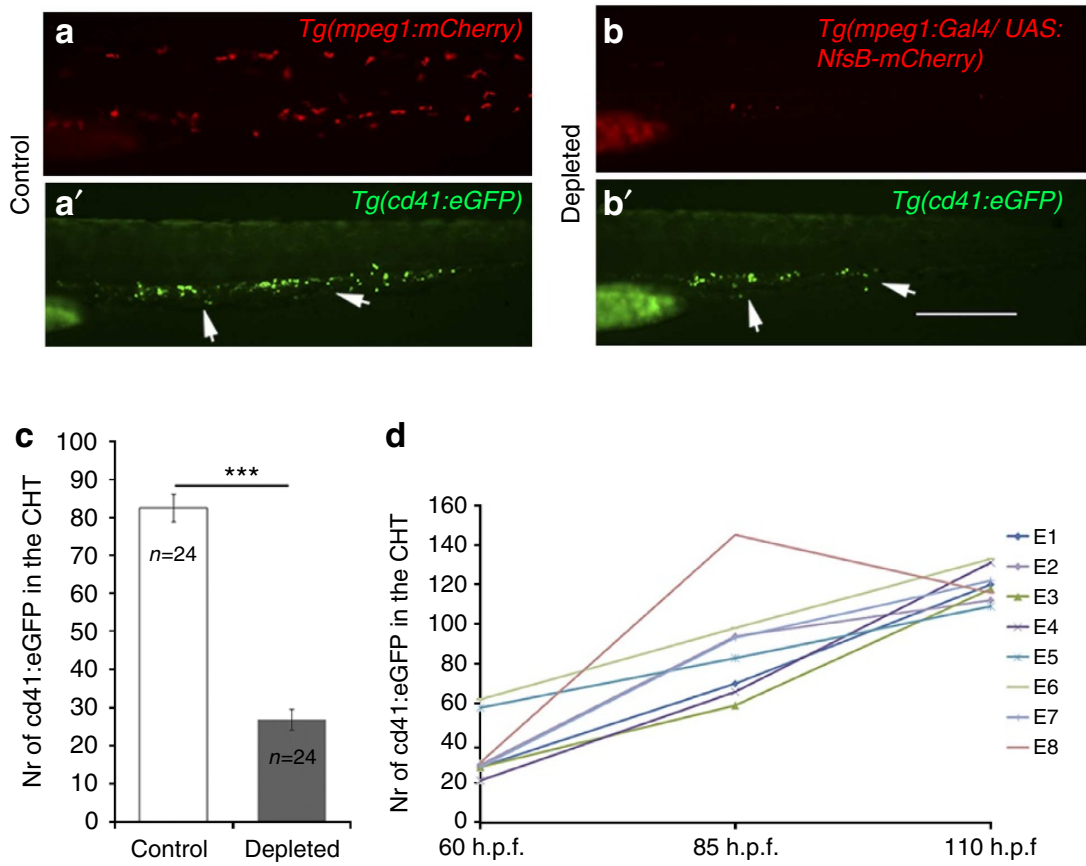

d

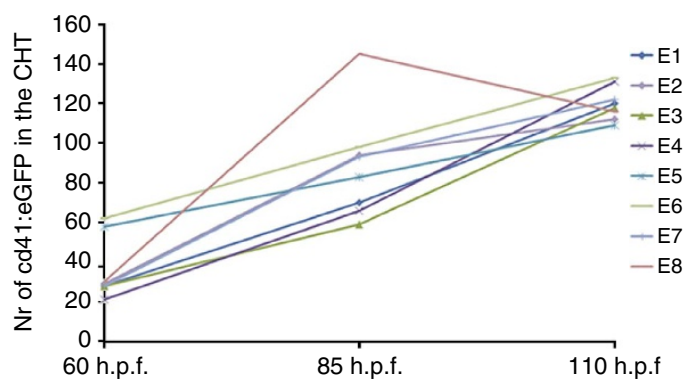

$\operatorname{Tg}$ (mpeg1:Gal4/ UAS: NfSB-mCherry)
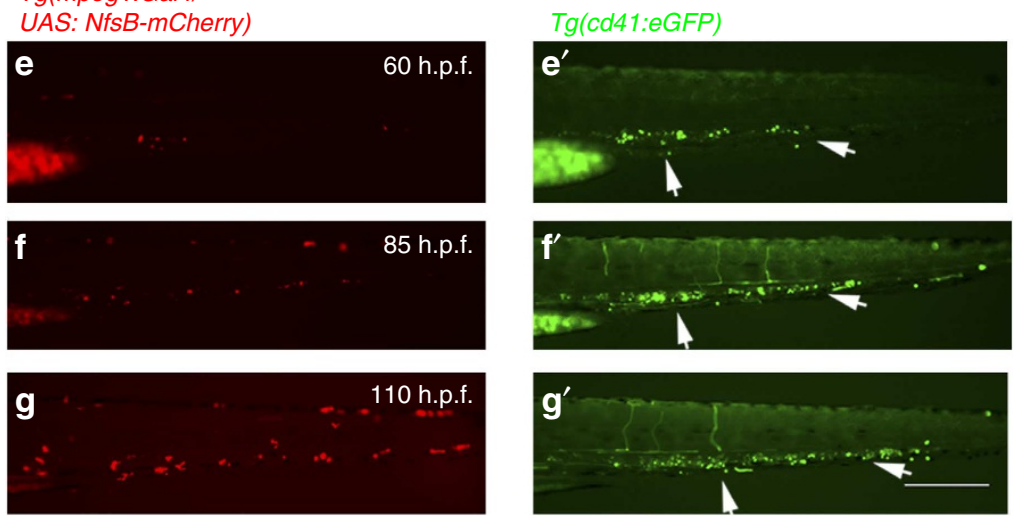

Figure 3 | Rescue of CHT colonization after macrophage reappearance. (a,b') Metronidazole induced depletion of macrophages (a,b) results in the decrease of $\mathrm{CHT}$ colonization $\left(\mathbf{a}^{\prime}, \mathbf{b}^{\prime}\right)$. (c) Metronidazole induced macrophage depletion substantially reduces HSPC/cd41:eGFP + colonization of the CHT ( $-68 \%)$ at 60 h.p.f. compared with the control Tg(cd41:eGFP//mpeg1:mCherry) embryos treated with metronidazole. Data are shown as average \pm s.e.m. $\star \star \star P<0.001$ (Wilcoxon test), $n=$ number of embryos of minimal three independent experiments. (d) Rescue of $\mathrm{CHT}$ colonization is held due to metronidazole withdrawal at 60 h.p.f. Graph represents number of HSPCs/cd41:eGFP + accumulated in the CHT at 60, 85 and 110 h.p.f. Data are shown as longitudinal study of single embryo, $n=8$. (e-g) Reappearance of macrophages is detected after the metronidazole wash-out during the following 2 days (25 (f) and $50 \mathrm{~h}$ post withdrawal $(\mathbf{g})$ ). The increasing number of HSPCs/cd41:eGFP + accumulated in CHT ( $\mathbf{e}^{\prime}-\mathbf{g}^{\prime}$ ) is directly connected to reappearance of macrophages in the embryo $(\mathbf{e}-\mathbf{g})$. Scale bar, $250 \mu \mathrm{m}$. See also Supplementary Fig. 3. 
macrophages. To study the role of MMPs, $T g(c d 41: e G F P)$ embryos were soaked from 38 h.p.f. in two different MMP inhibitors; GM6001, a general MMP inhibitor ${ }^{28}$ or SB-3CT, a specific inhibitor of MMP-2 and 9 (Gelatinases A and B $)^{29}$. This inhibition resulted in HSPCs/cd41:eGFP + accumulation in the AGM at 60 h.p.f. (Fig. 4 e,f and Supplementary Fig. $4 d,+26 \%$ and $+51 \%$ for GM6001 and SB-3CT respectively) compared with control embryos (Fig. 4d), while it led to a consequent decrease in HSPCs/cd41:eGFP colonization of the CHT at 60 h.p.f. (Fig. 4a-c; -43 and $-66 \%$ respectively, Supplementary Fig. $4 d, n=42$ and $n=44$ ) and the thymus at 72 h.p.f. (Supplementary Fig. $4 \mathrm{a}-\mathrm{d}$, -44 and $-75 \%$; respectively, $n=30$ and 29 ).

We thus concluded that both primitive macrophages and Mmps secretion are essential for HSPC mobilization and consequently haematopoietic organ colonization.

Mmp-9 and $m m p-13 a$ are expressed by macrophages. To establish a direct connection between macrophages and $m m p s$ expression in the AGM, we combined L-plastin immunostaining (a leukocyte marker) with WISH of 48 h.p.f. embryos using $m m p-$ 2, $m m p-9$ and $m m p-13 a$ probes. Mmp-9 and $-13 a$ expression patterns (Fig. $4 \mathrm{~g}-\mathrm{j}$ ) matched to myeloid cells (Fig. $4 \mathrm{~g}^{\prime}-\mathrm{h}^{\prime \prime}, n=10$ ) but not $m m p-2$ expression (Supplementary Fig. 4e,f, $n=10$ ), which gave a broader pattern in the trunk region (Supplementary Fig. $4 \mathrm{e}-\mathrm{e}^{\prime \prime}$ ).

As L-plastin is expressed by different myeloid populations, we analysed $m m p s$ expression in embryos injected with L-clodronate. We observed in the AGM that $m m p-2$ expression profile (Supplementary Fig. 4g,g') was unchanged after macrophage depletion while $m m p-13 a$ (Figs $41, \mathrm{l}^{\prime}$ ) was significantly reduced and $m m p-9$ completely disappeared (Fig. $4 \mathrm{k}, \mathrm{k}^{\prime}$ ).

We concluded that macrophages expressing $m m p-9$ are unambiguously associated with HSPC mobilization and intravasation.

Macrophage-mediated ECM degradation. WISH showed that $m m p-9$ is expressed by primitive macrophages, but no direct demonstration was made of macrophage-mediated matrix degradation. We therefore decided to visualize matrix degradation by in vivo zymography ${ }^{30}$ using gelatin as an intermediate product of collagen degradation. We injected highly quenched fluorescein-labelled gelatin (Gelatin-FITC) into the muscles of Tg(mpeg1:mCherry) embryos (Fig. 5a, $n=10$ ). Degradation of this substrate releases FITC from its quencher resulting in green fluorescence. Specific punctiform green fluorescence was observed around spherical cells that appeared as unlabelled ghosts (Fig. 5b,c, blue dots and arrow), while embryos soaked in MMP inhibitors did not show any FITC signal fluorescence (Supplementary Fig. 5a-c, middle and right, Supplementary Movie 5) nor did L-clodronate injected embryos (Supplementary Fig. 5d,e). Time-lapse imaging showed co-localization of macrophage trails and emitted FITC fluorescence (Fig. $5 c^{\prime}-f, j-$ m, Supplementary Movies 3-4 and Supplementary Fig. 5f, $n=3$, $79 \%$ of liberated FITC was correlated to macrophages). Figure $5 \mathrm{i}$ provides a schematic diagram of a macrophage migrating around the HSPC and leaving a trail of degraded gelatin (Fig. $5 c^{\prime}-h$, Supplementary Movie 3), followed by the migration and intravasation of a round cell between two spots of degraded gelatin (Fig. 5f-h, arrow; Supplementary Movie 3). To confirm

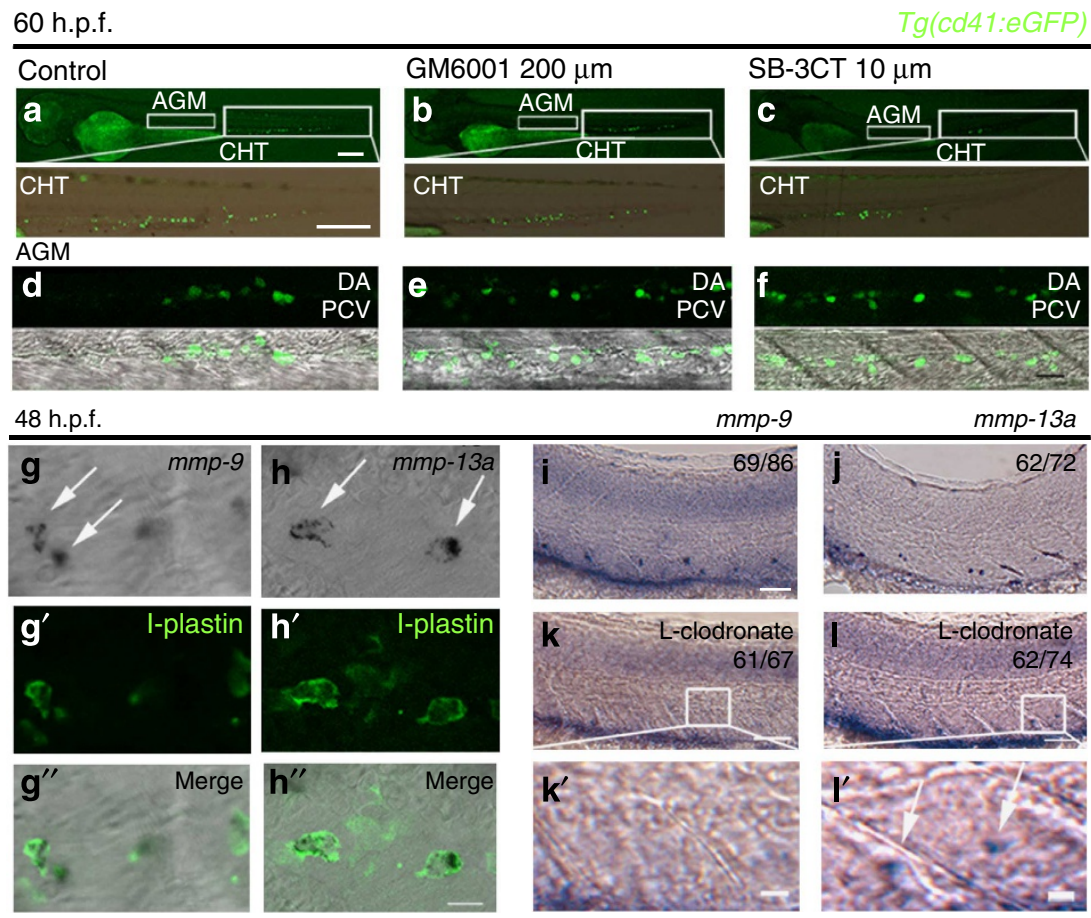

Figure 4 | Role of MMPs in HSPC mobilization and colonization of the CHT. (a-f) Inhibition of MMPs impairs HSPC mobilization and the colonization of CHT. (a) HSPCs are present in $\mathrm{Tg}(\mathrm{cd} 41 \mathrm{e}$ eGFP) embryo at 60 h.p.f. (bottom, enlarged CHT). Small box shows the AGM imaged by confocal microscopy in d. (d) Green fluorescence shows the presence of cd41:eGFP cells in the AGM at 60 h.p.f. (bottom, merged with transmission channel). (b,c) Treatment with GM6001 (b, $n=42)$ or SB-3CT (c, $n=44)$ reduces CHT colonization by HSPCs/cd41:eGFP + cells compared with control (a, $n=45)$. (e,f) Confocal imaging of the AGM reveals accumulation of HSPCs/cd41:eGFP + cells in AGM compared with the control (d) (d-f, $n=23$; three frames combined). Confocal imaging of WISH (g,h, arrows) combined with L-plastin immunofluorescence (green) ( $\mathbf{g}^{\prime}, \mathbf{h}^{\prime}$ ) are merged in $\mathbf{g}^{\prime \prime}$ and $\mathbf{h}^{\prime \prime}$. (i-I) WISH of mmp-9 (i) and $m m p-13 a(\mathbf{j})$ in the AGM in control embryos and after macrophage depletion by L-clodronate (k,I). Close-ups of macrophage depleted embryos in $\mathbf{k}^{\prime}, \mathbf{I}^{\prime}$ (arrow). Scale bar, $250 \mu \mathrm{m}(\mathbf{a}-\mathbf{c}), 30 \mu \mathrm{m}(\mathbf{d}-\mathbf{f}), 50 \mu \mathrm{m}(\mathbf{i}-\mathbf{I}), 10 \mu \mathrm{m}\left(\mathbf{g}, \mathbf{h}^{\prime \prime}, \mathbf{k}^{\prime}, \mathbf{I}^{\prime}\right)$. See also Supplementary Fig. 4. 


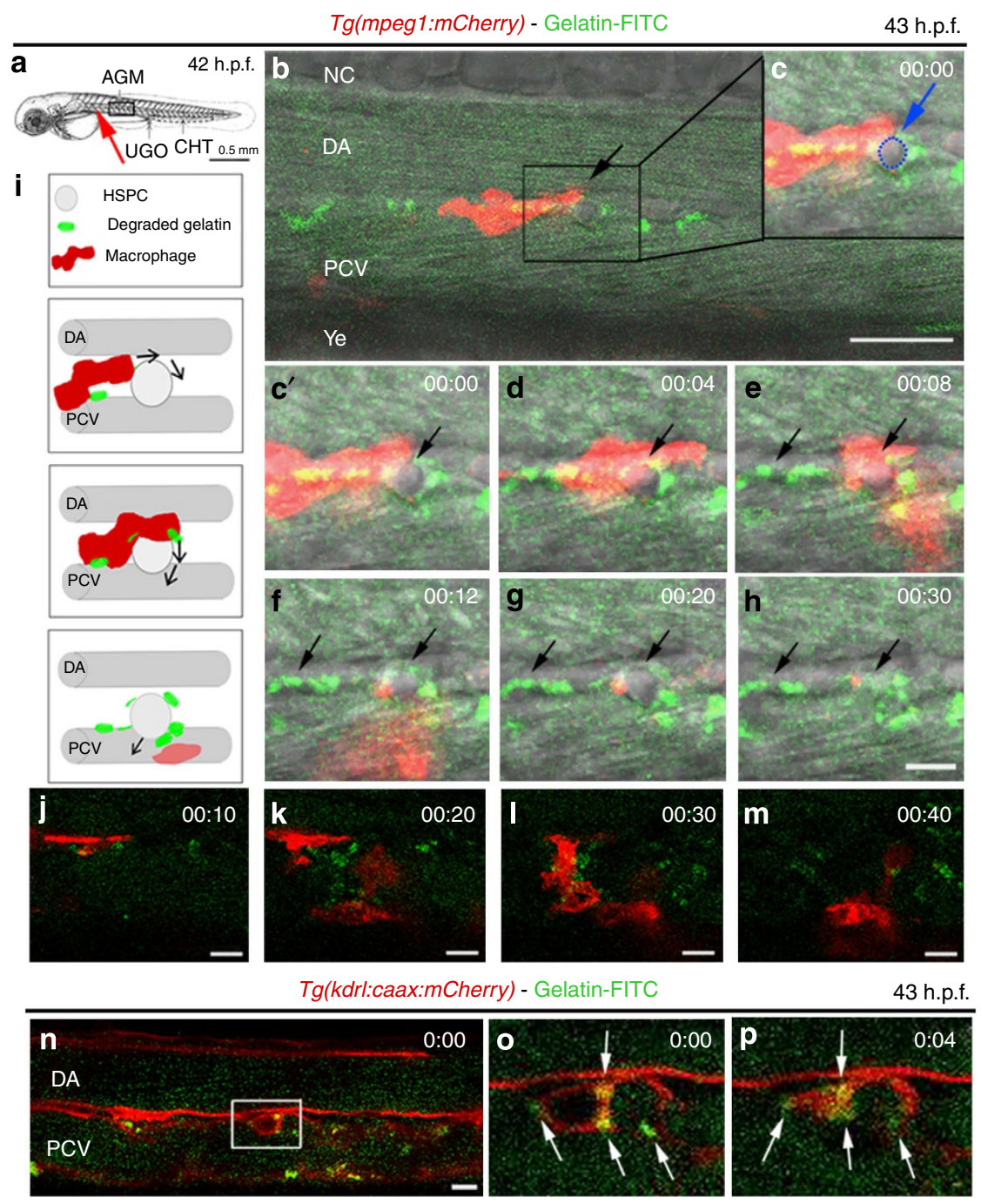

Figure 5 | In vivo zymography shows macrophage-induced proteolysis of the ECM. (a) Drawing indicates the site of intramuscular injection of GelatinFITC (red arrow) and the area imaged in $\mathbf{b}$ (black box). (b-h) In vivo imaging of the AGM at 43 h.p.f. was carried out just after gelatin-FITC injection in Tg(mpeg1:mCherry). Green staining indicates gelatin degradation after macrophage migration (red) in $\mathbf{c}-\mathbf{h}(n=10)$. (c-h) Close-ups (b, black box) show a patrolling macrophage (red) that degrades gelatin (green dots), induces HSPC migration through the ECM (arrow), followed by intravasation (Supplementary movie 3). (c) Arrow shows unlabelled round cells corresponding to HSPCs (blue dots). (i) Schematic view of gelatin degradation by a red macrophage around a light gray HSPC in the AGM. First diagram shows macrophage migration around an HSPC. Macrophage migration results in green spots corresponding to gelatin degradation, followed by HSPC intravasation (f-h, Supplementary Movie 3). (j-m) Zoomed imaging shows two macrophages in the AGM-degrading Gelatin-FITC during their passage (Supplementary Movie 4). (n) Gelatin degradation is localized around an emerging HSPC/mCherry + cell after injection of gelatin-FITC in a Tg(kdrl:caax:mCherry) embryo. (o,p) Close-ups of emerging HSPC/mCherry + intravasation in the vein (inset, $\mathbf{n}$ ) after ECM degradation in $4 \mathrm{~min}$. Time code in hours and minutes, upper right corner. White arrows point the FITC liberation next to the intravasating HSPC/mCherry + cells. UGO, urogenital opening. Scale bars, $0.5 \mathrm{~mm}$ (a), $30 \mu \mathrm{m}$ (b) and $10 \mu \mathrm{m}\left(\mathbf{c}^{\prime}-\mathbf{n}\right)$. See also Supplementary Fig. 5 and Supplementary Movies 3-5.

that these migrating round cells are HSPCs, we injected GelatinFITC into $\mathrm{Tg}(k d r l: c a a x: m C h e r r y)$ embryos in which vascular endothelium and HSPCs express the mCherry protein ${ }^{10}$. We observed the same specific punctiform fluorescence around HSPCs/mCherry + cells emerging from the wall of the aorta (Fig. $5 \mathrm{n}-\mathrm{p}, n=4$ ).

In vivo zymography therefore provided a final demonstration of matrix degradation in the AGM by macrophage secreted proteases followed by HSPC mobilization and vein intravasation.

\section{Discussion}

The studies described herein show an unexpected accumulation of primitive macrophages in the AGM region (ventrally to the DA) of human and zebrafish embryo.
We previously reported that zebrafish HSPCs emerge in the subaortic mesenchyme then enter the PCV circulation through peculiar structures, which facilitate HSPC intravasation'. Live imaging in HSPC-cd41:eGFP +/macrophage-mpeg1:mCherry + zebrafish embryo shows these tiny entry points are created by macrophages during their migration through the stroma, which will allow HSPCs to join the PCV.

Macrophage depletion at early stages of development (25 h.p.f.) prevents the colonization of haematopoietic organs without affecting EHT in the AGM, while late depletion (45 h.p.f.) leads to less pronounced effects. Because zebrafish definitive haematopoiesis occurs between 30 and 55 h.p.f. (ref. 3), the partial colonization observed after late depletion is likely due to HSPCs which had already left the AGM before macrophage depletion. 
a
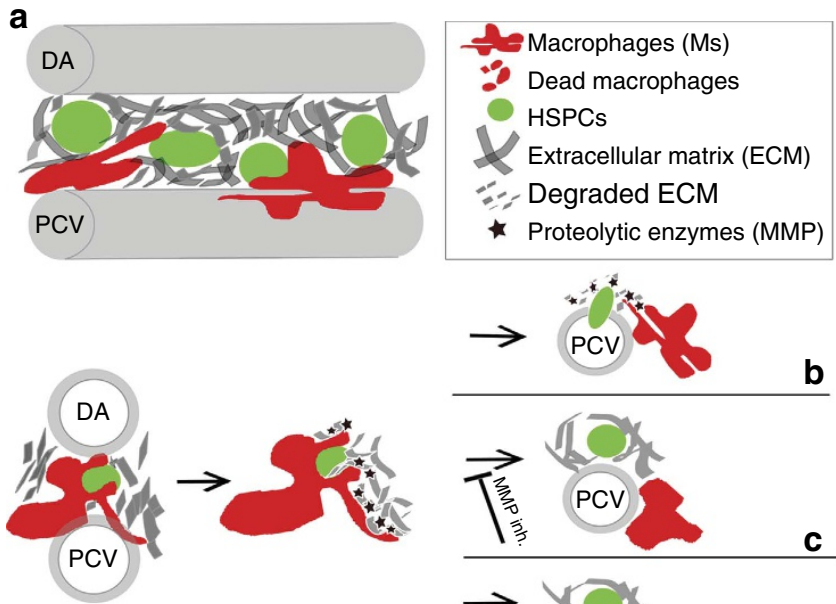

b
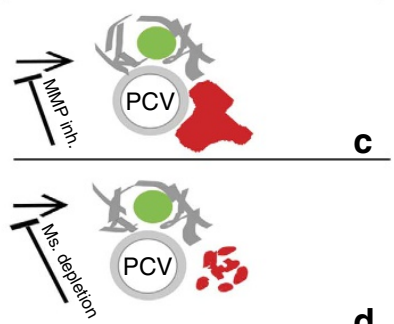

C

Figure 6 | Primitive macrophages in HSPC mobilization and definitive

haematopoiesis. (a) Macrophages (red) interact with HSPCs (green) in the AGM during haematopoiesis. (b) ECM degradation by macrophages enables HSPC mobilization, intravasation and haematopoietic organ colonization. Mmps inhibition (c) or macrophage depletion (d) result in HSPC accumulation in the AGM. inh., inhibition.

We show here that these entry points are formed by Mmpssecreting macrophages. Specific chemical Mmps inhibition together with combined WISH and immunofluorescence staining established that macrophages are involved in HSPC mobilization via Mmp-9 secretion.

Therefore, our data suggest that primitive macrophages play an essential role in definitive haematopoiesis by inducing Mmpsmediated degradation of the ECM around HSPC in the AGM region. Matrix degradation allows mobilization and migration of HSPC into the stroma, driving their intravasation into the vein, leading ultimately to the colonization of haematopoietic organs and the establishment of definitive haematopoiesis (as summarized in Fig. 6).

Another intriguing observation of this report is that a specific accumulation of primitive macrophages is also detected in the human embryo. CD68 + macrophages are indeed concentrated in the mesenchyme beneath the ventral wall of the human embryonic aorta but also associated with the intra-aortic HSPC clusters. Even if, in the human, like any mammalian embryo the mobilization of HSPCs occurs within the DA circulation ${ }^{1}$, this accumulation of macrophages observed in the AGM, suggests that they could play a role in the establishment of definitive haematopoiesis also in these species. Therefore, we speculate that in human embryo, macrophages can facilitate the release of HSPCs from intra-aortic clusters, through a process evolutionary conserved.

Recent findings have demonstrated that macrophages maintain HSCs in adult bone marrow niches and their depletion mobilizes HSPCs in the bloodstream ${ }^{31,32}$. Our data show that during development, macrophages may have the opposite function by mobilizing HSPCs from the AGM followed by their intravasation and colonization of haematopoietic organs. This mobilization involves ECM degradation by the local secretion of Mmps.

Recent studies have shown that tumour-associated macrophages play a role in ECM remodelling in tumour stroma through the release of proteases from invadopodia, (mainly MMP-2 and 9) (ref. 18,19). These enzymes degrade the rigid stroma of tumours and enable CICs (cancer-initiating cells) to intravasate and seed secondary sites, that is, metastasis ${ }^{33}$. Our observations show marked similarities with this process, suggesting that HSPC and CIC migration might be comparable. It will thus be interesting to explore whether HSPCs also release factors that attract macrophages and what are their nature.

\section{Methods}

Human tissues and immunostaining. Human embryos were obtained from voluntary abortions performed according to guidelines and with the approval of the French National Ethics Committee. Patients gave their written consent for the embryos to be used for research purposes. Embryo sections $(5 \mu \mathrm{m})$ were incubated with anti-CD34 $\left(3.2 \mu \mathrm{g} \mathrm{ml}^{-1}\right.$, Miltenyi) or anti-CD68 $\left(10 \mu \mathrm{g} \mathrm{ml}^{-1}\right.$, eBioscience) antibodies, then with biotinylated secondary antibodies $\left(10 \mu \mathrm{g} \mathrm{ml}^{-1}\right.$, Immunotech) and with peroxidase-labelled streptavidin $\left(0.6 \mu \mathrm{g} \mathrm{ml}^{-1}\right.$, Immunotech). Fluorescence-conjugated secondary antibodies $\left(0.5 \mu \mathrm{g} \mathrm{ml}^{-1}\right.$, Biolegend) were used for double staining.

Zebrafish transgenic lines and live imaging. Wild-type $\mathrm{AB}, \mathrm{Tg}(\mathrm{kdrl}: \mathrm{Has}$. HRAS-mCherry) (here cited as kdrl:caax:mCherry) ${ }^{34}, \operatorname{Tg}(c d 41: e G F P)^{35}$,

$\mathrm{Tg}(m p x: e G F P)^{36}, \mathrm{Tg}(m p e g 1: m C h e r r y-f)$ (here cited as mpeg1:mCherry; see below) and Tg(mpeg1:Gal4FF//UAS-E1b:Eco.NfsB-mCherry) (here cited as mpeg1: GAL4//UAS:NfsB-mCherry) ${ }^{26}$ transgenic embryos were staged as described by Kimmel et al. ${ }^{37}$ All experiments were carried out following the protocol CEEA-LR13007 approved by the Animal Care and Use Committee Languedoc-Roussillon.

Human and zebrafish embryos (lateral views, rostral to the left) were imaged on Leica SP5 confocal (Fig. 1a-b" ${ }^{\prime \prime}$, Fig. 5, Supplementary Fig. 1 and Supplementary Movies 3 and 4), Leica SPE confocal (Fig. 1c; Fig. 4d,g-h" and Supplementary Fig. 4a-c, e- $e^{\prime \prime}$, Supplementary Fig. 5d,e), DeltaVision OMX (Applied Precision; Figs 1e-1 and Supplementary Movie 1), Zeiss LSM510 confocal (Fig. 2a,b, Supplementary Fig. 5a-c, Supplementary Movies 2 and 5), Zeiss V12 (Fig. 2c,d, Fig. $3 \mathrm{a}-\mathrm{b}^{\prime}$ and $\mathrm{e}-\mathrm{g}^{\prime}$ ), Trophos Plate Runner (Figs 2e-m), Olympus MVX10 (Fig. $4 \mathrm{a}-\mathrm{c}$ and Supplementary Fig. 2a-f) and Zeiss AxioImager Z.1 (Fig. 4i- $\mathrm{l}^{\prime}$ and Supplementary Fig. $4 \mathrm{f}-\mathrm{g}^{\prime}$ ).

Generation of mpeg1:mCherry-f transgenic Zebrafish line. A 1.9-kb mpeg1 promoter fragment encompassing the mpeg sequences driving promoter activity specifically in macrophages ${ }^{38}$ was amplified by PCR using following forward $5^{\prime}$-TTGGAGCACATCTGAC- $3^{\prime}$ and NotI-introducing reverse $5^{\prime}$-TTATAGCGG CCGCGAAATGCTCTTGACTTCATGA- $3^{\prime}$ primers. Resulting product was digested by NotI and ligated to farnesylated mCherry protein coding sequence so that Mpeg1 ATG initiation codon would be in frame with downstream mCherry-F cds in a Tol 2 derived vector. The resulting plasmid was injected along with transposase mRNA in one cell stage zebrafish embryos to achieve germline integration.

Macrophage chemical and inducible genetic depletion. Macrophages were depleted by injecting $5 \mathrm{nl}$ of L-clodronate into the caudal vein (gift from M. Daeron Laboratory). L-PBS was injected as a control. Embryos with L-clodronate aggregates causing vessel occlusions and shunts were discarded.

Metronidazole-mediated depletion was performed by using double transgenic fish $\mathrm{Tg}$ (mpeg1:GAL4//UAS:NfsB-mCherry $)^{26}$ crossed with $\operatorname{Tg}(c d 41: e G F P)$ to evaluate the effect of macrophage depletion on cd41+ cells. Embryos at 18 h.p.f. were treated with freshly prepared $10 \mathrm{mM}$ metronidazole (Sigma) in $0.1 \%$ DMSO (dimethylsulphoxide) solution protected from light until the evaluation (60 h.p.f.), then rinsed with embryo water. $\mathrm{Tg}(\mathrm{cd}$ 41:eGFP//mpeg1:mCherry) embryos treated with $10 \mathrm{mM}$ metronidazole were used as control.

MMP inhibition assay. Embryos were soaked in GM6001 (200 $\mu$ M, Merck Millipore), SB-3CT (10 $\mu \mathrm{M}$, Enzo Life Sciences) or DMSO $(0.2 \%)$ as a control from 38 h.p.f. until observation.

WISH and immunofluorescence staining. WISH was performed according to Thisse (http://zfin.org/zf_info/zfbook/chapt9/9.82.html). Embryos at 48 h.p.f. were fixed, dehydrated with methanol, digested with proteinase $\mathrm{K}$ (New England Biolabs) and fixed again. Prehybridization was performed at $68^{\circ} \mathrm{C}$ in $\mathrm{HM}+$ buffer ( $50 \%$ formamide, $5 \times$ saline sodium citrate, $0.1 \%$ Tween- $20,50 \mu \mathrm{g} \mathrm{ml}^{-1}$ heparin, $500 \mu \mathrm{g} \mathrm{ml}^{-1}$ yeast tRNA) and followed by hybridization with $0.5 \mathrm{ng}^{-1} \mathrm{l}^{-1}$ digoxigenin-labelled antisense RNA probe in $\mathrm{HM}+$ buffer. The probes were detected with alkaline phosphatase-conjugated anti-digoxigenin antibodies $(1 / 5,000$; Roche Applied Science) using nitro blue tetrazolium chloride/5-bromo-4-chloro-3-indolyl-phosphate, toluidine-salt (Roche Applied Science). After staining embryos were mounted in $90 \%$ glycerol prior imaging.

Zebrafish Mmp-9 and 13a plasmids were gifts from L. Ramakrishnan laboratory, mmp-2 from A. Noel laboratory. For immunostaining, embryos were incubated with anti-L-plastin antibody (1/10,000, gift from M. Redd), followed by Alexa Fluor 488 Goat Anti-Rabbit IgG antibody (1/1,000, Life Technologies). 
In vivo zymography. In vivo zymography was performed according to Crawford's protocol $^{30}$. FITC conjugated gelatin (Gelatin-FITC, Anaspec) $1 \mathrm{mg} \mathrm{ml}^{-1}$ in PBS was injected ( $4-8 \mathrm{ng}$ ) into muscle between somite 4 and 5 at 42 h.p.f. Imaging was performed just after injections. Control embryos were either incubated with MMP inhibitors during $4 \mathrm{~h}$ prior injections or injected with L-clodronate $17 \mathrm{~h}$ prior Gelatin-FITC injections.

Statistical analysis. Normal distributions were analysed using Shapiro-Wilk test. Non-gaussian data were analysed using Wilcoxon or Kruskal-Wallis test, Gaussian with Student's $t$-test or analysis of variance followed by Holm's multiple comparison. $P<0.05$ was considered as statistically significant (symbols: ${ }^{* * *} P<0.001$; $\left.{ }^{*} P<0.01 ;{ }^{*} P<0.05\right)$. Statistical analyses were performed using $\mathrm{R}$ software.

\section{References}

1. Tavian, M., Biasch, K., Sinka, L., Vallet, J. \& Péault, B. Embryonic origin of human hematopoiesis. Int. J. Dev. Biol. 54, 1061-1065 (2010).

2. Cumano, A. \& Godin, I. Ontogeny of the hematopoietic system. Annu. Rev. Immunol. 25, 745-785 (2007).

3. Murayama, E. et al. Tracing hematopoietic precursor migration to successive hematopoietic organs during zebrafish development. Immunity 25, 963-975 (2006).

4. Mikkola, H. K. a. \& Orkin, S. H. The journey of developing hematopoietic stem cells. Development 133, 3733-3744 (2006).

5. Guyader, D. L. e. et al. Origins and unconventional behavior of neutrophils in developing zebrafish origins and unconventional behavior of neutrophils in developing zebrafish. Blood 111, 132-141 (2008).

6. Herbomel, P., Thisse, B. \& Thisse, C. Ontogeny and behaviour of early macrophages in the zebrafish embryo. Development 126, 3735-3745 (1999).

7. Gering, M., Rodaway, A. R., Göttgens, B., Patient, R. K. \& Green, A. R. The SCL gene specifies haemangioblast development from early mesoderm. EMBO J. 17, 4029-4045 (1998).

8. Gering, M. \& Patient, R. Notch signalling and haematopoietic stem cell formation during embryogenesis. J. Cell Physiol. 222, 11-16 (2005).

9. Kissa, K. et al. Live imaging of emerging hematopoietic stem cells and early thymus colonization. Blood 111, 1147-1156 (2008).

10. Bertrand, J. Y. et al. Haematopoietic stem cells derive directly from aortic endothelium during development. Nature 464, 108-111 (2010).

11. Kissa, K. \& Herbomel, P. Blood stem cells emerge from aortic endothelium by a novel type of cell transition. Nature 464, 112-115 (2010).

12. Boisset, J.-C. et al. In vivo imaging of haematopoietic cells emerging from the mouse aortic endothelium. Nature 464, 116-120 (2010).

13. Oberlin, E., Tavian, M., Blazsek, I. \& Péault, B. Blood-forming potential of vascular endothelium in the human embryo. Development 129, 4147-4157 (2002).

14. Wynn, T. a., Chawla, A. \& Pollard, J. W. Macrophage biology in development, homeostasis and disease. Nature 496, 445-455 (2013).

15. Mantovani, A., Biswas, S. K., Galdiero, M. R., Sica, A. \& Locati, M. Macrophage plasticity and polarization in tissue repair and remodelling. J. Pathol. 229, 176-185 (2013)

16. Lin, E. Y., Nguyen, A. V., Russell, R. G. \& Pollard, J. W. Colony-stimulating factor 1 promotes progression of mammary tumors to malignancy. J. Exp. Med. 193, 727-740 (2001).

17. Pollard, J. W. Tumour-educated macrophages promote tumour progression and metastasis. Nat. Rev. Cancer 4, 1-8 (2004).

18. Linder, S. The matrix corroded: podosomes and invadopodia in extracellular matrix degradation. Trends Cell Biol. 17, 107-117 (2007).

19. Vérollet, C. et al. Extracellular proteolysis in macrophage migration: losing grip for a breakthrough. Eur. J. Immunol. 41, 2805-2813 (2011).

20. Kessenbrock, K., Plaks, V. \& Werb, Z. Matrix metalloproteinases: regulators of the tumor microenvironment. Cell 141, 52-67 (2010).

21. Yoong, S. et al. Characterization of the zebrafish matrix metalloproteinase 9 gene and its developmental expression pattern. Gene Expr. Patterns 7, 39-46 (2007).

22. Qian, F. et al. Microarray analysis of zebrafish cloche mutant using amplified cDNA and identification of potential downstream target genes. Dev. Dyn. 233, 1163-1172 (2005)

23. Tavian, M., Hallais, M. F. \& Péault, B. Emergence of intraembryonic hematopoietic precursors in the pre-liver human embryo. Development 126, 793-803 (1999).
24. Bernut, A. et al. Mycobacterium abscessus cording prevents phagocytosis and promotes abscess formation. Proc. Natl Acad. Sci. USA 111, E943-E952 (2014)

25. Mancardi, D. a. et al. The high-affinity human IgG receptor Fc $\gamma \mathrm{RI}$ (CD64) promotes IgG-mediated inflammation, anaphylaxis, and antitumor immunotherapy. Blood 121, 1563-1573 (2013).

26. Palha, N. et al. Real-time whole-body visualization of Chikungunya Virus infection and host interferon response in zebrafish. PLoS Pathog. 9, e1003619 (2013).

27. Zhang, J., Bai, S., Zhang, X., Nagase, H. \& Sarras, M. P. The expression of gelatinase A (MMP-2) is required for normal development of zebrafish embryos. Dev. Genes Evol. 213, 456-463 (2003).

28. Bai, S. et al. Matrix metalloproteinase expression and function during fin regeneration in zebrafish: analysis of MT1-MMP, MMP2 and TIMP2. Matrix Biol. 24, 247-260 (2005)

29. Krüger, A. et al. Antimetastatic activity of a novel mechanism-based gelatinase inhibitor. Cancer Res. 65, 3523-3526 (2005).

30. Crawford, B. D. \& Pilgrim, D. B. Ontogeny and regulation of matrix metalloproteinase activity in the zebrafish embryo by in vitro and in vivo zymography. Dev. Biol. 286, 405-414 (2005).

31. Winkler, I. G. et al. Bone marrow macrophages maintain hematopoietic stem cell (HSC) niches and their depletion mobilizes HSCs. Blood 116, 4815-4828 (2010).

32. Chow, A. et al. Bone marrow $\mathrm{CD} 169+$ macrophages promote the retention of hematopoietic stem and progenitor cells in the mesenchymal stem cell niche. J. Exp. Med. 208, 261-271 (2011).

33. Condeelis, J. \& Segall, J. E. Intravital imaging of cell movement in tumours. Nat. Rev. Cancer 3, 921-930 (2003).

34. Chi, N. C. et al. Foxn4 directly regulates tbx $2 \mathrm{~b}$ expression and atrioventricular canal formation. Genes Dev. 22, 734-739 (2008).

35. Lin, H.-F. et al. Analysis of thrombocyte development in CD41-GFP transgenic zebrafish. Blood 106, 3803-3810 (2005)

36. Renshaw, S. a. et al. A transgenic zebrafish model of neutrophilic inflammation. Blood 108, 3976-3978 (2006)

37. Kimmel, C. B., Ballard, W. W., Kimmel, S. R., Ullmann, B. \& Schilling, T. F. Stages of embryonic development of the zebrafish. Dev. Dyn. 203, 253-310 (1995).

38. Ellett, F., Pase, L., Hayman, J. W., Andrianopoulos, A. \& Lieschke, G. J. Mpeg1 promoter transgenes direct macrophage-lineage expression in zebrafish. Blood 117, e49-e56 (2011)

\section{Acknowledgements}

We thank V. Diakou, V. Georget and the MRI facility for their assistance; D. Mancardi and M. Daeron for the L-clodronate gift; A. Meijer for mpeg1:Gal4 fish; L. Ramakrishnan for mmp-9 and mmp-13 plasmids; A. Noel for mmp-2 plasmid; M. Redd for L-plastin antibody; and M. Rossel for discussions. We thank P. Herbomel for hosting the initial phase of this work at Institut Pasteur. This work was supported by ARC and FishForPharma (EU, FP7). J.T. was supported by a fellowship from the MESR, VTC by a fellowship from the DARRI, Institut Pasteur and by a fellowship from the Région Languedoc-Roussillon, Chercheur d'Avenir.

\section{Author contributions}

J.T., V.T.C., E.J., J.M.-L., E.L., M.T. and K.K. designed and performed the experiments. C.G. and G.L. generated the mpeg1:mCherry transgenic fish line. J.T., E.L. and K.K. wrote the manuscript with input from G.L. and M.T.

\section{Additional information}

Supplementary Information accompanies this paper at http://www.nature.com/ naturecommunications

Competing financial interests: The authors declare no competing financial interests

Reprints and permission information is available online at http://npg.nature.com/ reprintsandpermissions/

How to cite this article: Travnickova, J. et al. Primitive macrophages control HSPC mobilization and definitive haematopoiesis. Nat. Commun. 6:6227 doi: 10.1038/ ncomms7227 (2015). 